\title{
Expected impact of lockdown measures due to COVID-19 on disabling conditions: a modelling study of chronic low back pain
}

\author{
Fabio Galbusera' ${ }^{1} \cdot$ Pierre Côtè $\grave{2}^{2,3} \cdot$ Stefano Negrini ${ }^{1,4}$ \\ Received: 12 April 2021 / Accepted: 19 July 2021 / Published online: 27 July 2021 \\ (c) The Author(s), under exclusive licence to Springer-Verlag GmbH Germany, part of Springer Nature 2021
}

\begin{abstract}
Purpose The COVID-19 pandemic and the containment measures such as social distancing, mobility restrictions and quarantine have significantly impacted the delivery of healthcare services, with possible negative effects on low back pain patients. In this study, we used an innovative agent-based model to quantify the effects of COVID-19 on the prevalence and severity of low back pain in the general population.

Methods Epidemiological data were used to simulate the low back pain evolution in a population of 300,000 agents. Reduced access to treatment due to the containment measures was simulated with a probabilistic approach, in which 500 random scenarios (differing in: length of the lockdown, probability of having access to treatment, time before the resumption of treatment, duration of the effects of the treatment after its interruption) were simulated.

Results The lockdown may increase the mean pain score higher than 1/10 points for patients suffering from acute low back pain, up to 4-5/10 points for specific individuals. The lockdown also affected the length of pain episodes, possibly impacting chronicity and disability. All the variables describing the random scenarios had a relevant impact in determining both the increase of pain intensity in the population and the length of the effects of the lockdown.

Conclusions "Optimal lockdown parameters" which minimize the impact on low back pain while preserving the effects on infection spread and mortality could not be identified. Policies favouring a prompt resumption of treatments after the lockdown may be effective in shortening the duration of its negative effects.
\end{abstract}

Keywords Agent-based model $\cdot$ COVID-19 $\cdot$ Low back pain $\cdot$ Social distancing $\cdot$ Lockdown

\section{Introduction}

The COVID-19 pandemic has significantly impacted the delivery of all healthcare services. Containment measures such as social distancing, mobility restrictions and quarantine are having substantial short- and long-term consequences on patients suffering from non-communicable diseases. Specifically, non-urgent visits have been cancelled

Fabio Galbusera

fabio.galbusera@grupposandonato.it

1 IRCCS Istituto Ortopedico Galeazzi, Via Galeazzi 4, 20161 Milan, Italy

2 Faculty of Health Sciences, Ontario Technical University, Oshawa, ON, Canada

3 Center for Disability Prevention and Rehabilitation at Ontario Tech University and CMCC Oshawa, Oshawa, ON, Canada

4 Department of Biomedical, Surgical and Dental Sciences, University Degli Studi di Milano, Milan, Italy in many countries, patients with new complaints may not receive adequate diagnosis and treatment, and the progression of diseases may not be adequately monitored and managed $[1,2]$. It is plausible that the reduced access to health care services will lead to an increase in the burden of disability related to non-communicable diseases such as low back pain.

Low back pain (LBP) is the leading cause of disability globally and a significant public health problem [3-6]. Patients with restricted access to adequate treatment would likely experience worsening of their quality of life and increased disability during and after the lockdown. It is also likely that the lack of physical activity during the lockdown has increased disability related to LBP and delayed return to work after the lockdown. The impact of COVID-19 on disability has been recently discussed [7] but, to our knowledge, no study has explored the impact of the lockdown on LBP or modelled the effect of the measures adopted to contain the COVID-19 transmission on the course of LBP and disability. 
Mathematical models have been widely used to investigate the dynamics of populations and biological systems [8], such as for example the evolution of demographics, the interaction between predators and preys [9], and the spread of infections [10]. Among the various types of models which have been developed in the healthcare field, agent-based models have been successfully employed in many situations, including the study of epidemics [11], oncology [12], and inflammation [13]. To our knowledge, mathematical models aimed at investigating LBP in the population have never been reported.

In this study, we use an innovative simulation modelling approach to quantify the effects of the containment measures implemented during the COVID-19 pandemic on the prevalence and severity of LBP in the general population. Our population-based model also aimed to predict the distribution and severity of LBP in the general population, which could serve as a basis to understand the effects of the lockdown and assist with the development of post-lockdown rehabilitation interventions.

\section{Materials and methods}

\section{Patient and public involvement statement}

Patients or the public were not involved in the design, or conduct, or reporting, or dissemination plans of our research.

\section{Simulation of LBP in the population}

We used an agent-based model to simulate the evolution of LBP in the population $[14,15]$. The model included a number of agents, each representing one individual susceptible to develop LBP; a preliminary sensitivity study revealed that 300,000 agents were sufficient to capture the behaviour of the population, and using more agents would have a minimal impact on the results. We used epidemiological data of the incidence of LBP and simulated its evolution using precise real-world time horizon with increments of one day [16]. At the beginning of the simulation, each agent was randomly assigned an age, so that the simulated population replicated the age distribution of the population of the United States of America [17]. Life expectancy was randomly assigned to all agents based on the estimated year of birth [18], assuming a normal distribution and a standard deviation of 15 years [19]. At the end of its simulated life span, each agent was replaced by a new one with a different random life expectancy. This realistic implementation aimed at achieving a substantially constant age distribution of the simulated population over time. All agents with age greater than 18 were assumed to be at risk of developing LBP.
The incidence of LBP was derived from an epidemiological study of the general population [20], which reported annual incidence of $1 \%$ for intense episodes and $0.4 \%$ for disabling LBP. We focused on intense and disabling LBP because they are more likely to have healthcare visits than mild episodes [21]. The cumulative annual incidence of $1.4 \%$ was converted to a daily incidence of $0.0038 \%$; this number was used as the probability of randomly developing a LBP episode in susceptible individuals in each day of the simulation.

At the beginning of the simulation, we assumed that no individuals had LBP or had previous LBP episodes in the previous year. The first stages of the simulation, therefore, modelled a transient behaviour which ended when LBP prevalence and average pain scores showed negligible changes (less than $0.1 \%$ ) with progressing time; such steady state was reached after simulating 35 years. We then simulated the suspension of treatment (lockdown) starting at the end of the transient phase when the prevalence of LBP was realistically distributed in the population.

Each incident episode of LBP was assigned to one of five trajectories used to describe the evolution of LBP: (1) rapid pain recovery, (2) recovery by week 12 , (3) incomplete pain recovery/persistent mild pain, (4) fluctuating pain, (5) persistent high pain [22]. The assignment was random and based on published values of the incidence of each cluster: cluster $1,35.8 \%$; cluster $2,34.3 \%$; cluster $3,14 \%$; cluster $4,10.5 \%$, cluster $5,5.4 \%$ [22]. In case of individuals older than 45 years or those with previous LBP episodes, new episodes had a higher probability to belong to cluster 4 (fluctuating pain) to reflect the higher odds ratio reported in the literature [22]. In this case, the probabilities were adjusted to: cluster $1,31.6 \%$; cluster 2 , $30.3 \%$; cluster $3,12.4 \%$; cluster $4,21 \%$, cluster $5,4.7 \%$. For each LBP episode, the daily level of pain was randomly selected based on the mean values and standard deviations reported in the literature [22], assuming a normal distribution in the population (Fig. 1).

Individuals assigned to clusters 1 and 2 had their episodes terminated at the end of the 12th week, or randomly assigned to become persistent mild pain for a duration of at least one year (Fig. 2). For agents in clusters 3-5 the pain episode continued for at least one year from the date of onset following the relative trajectory. At the end of the simulated year, one of three possible outcomes was randomly selected: (1) termination of the pain episode; (2) transition to another cluster; or (3) continuation in the same cluster for another year. The probabilities of changing cluster or of terminating the LBP episode were estimated based on posterior probability values for cluster membership determined in a long-term study [23] (Table 1). 

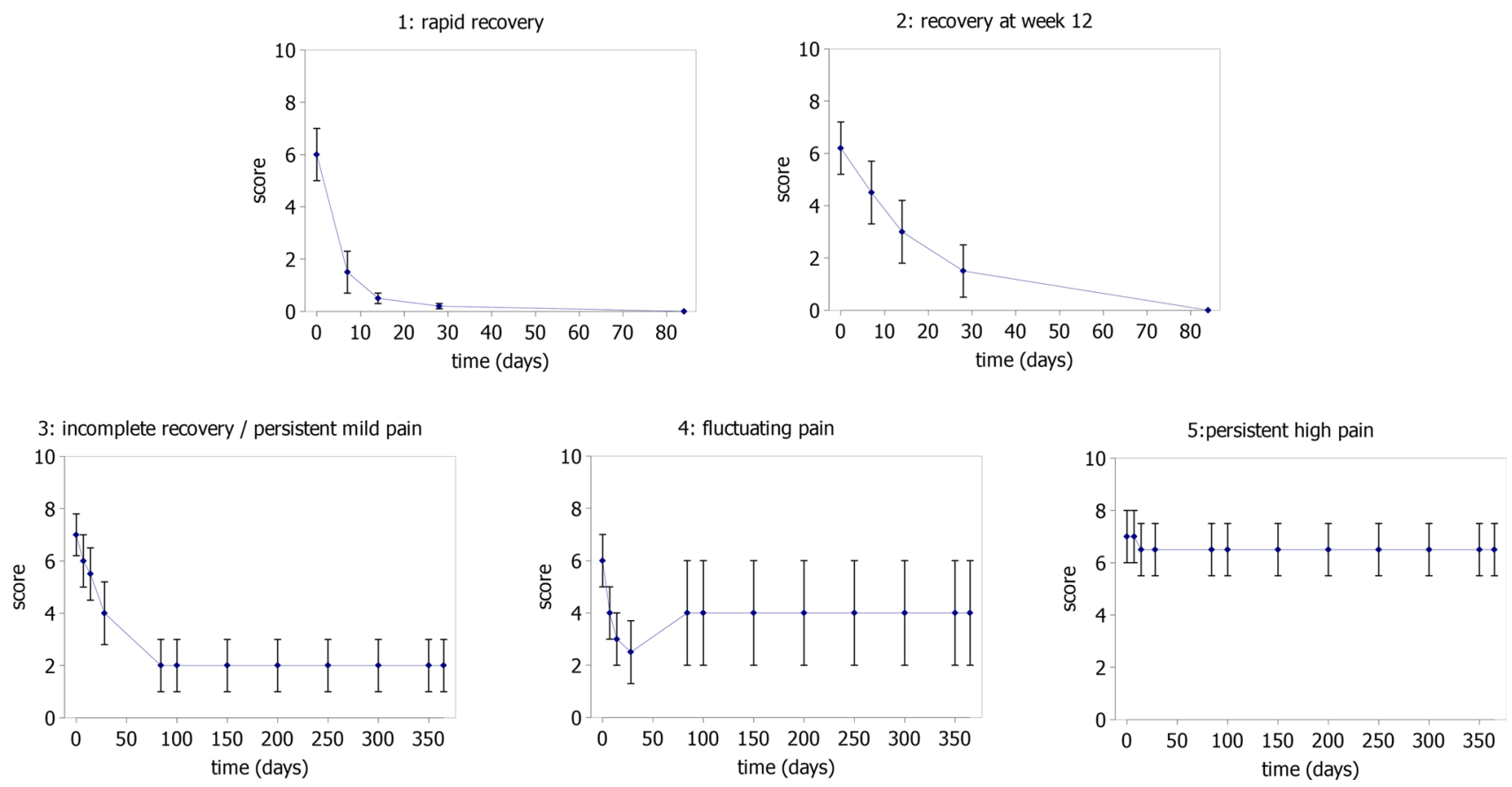

Fig. 1 Mean values and standard deviations of the pain for the trajectories associated with the five clusters: 1, rapid recovery; 2, recovery at week 12; 3, incomplete recovery/persistent mild pain; 4: fluctuating pain; 5: persistent high pain. Data derived from [22]

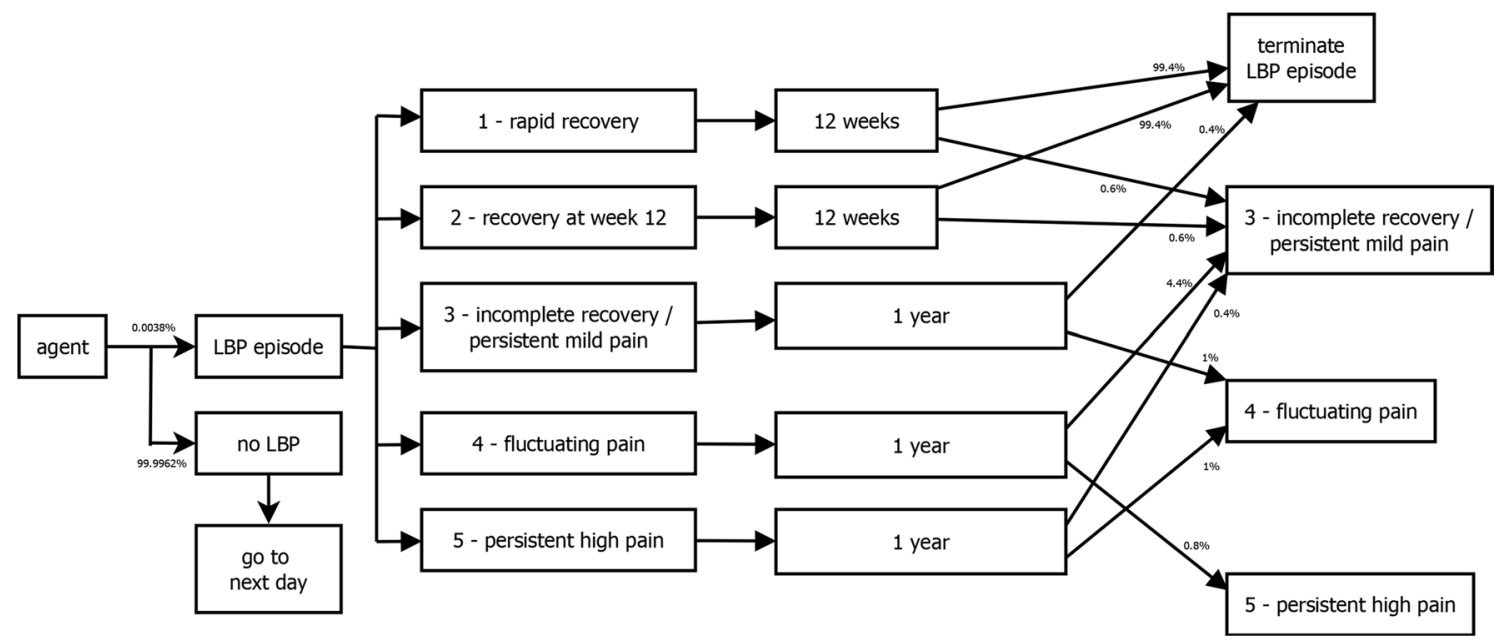

Fig. 2 Schematic representation of the behaviour of one susceptible agent, indicating the probabilities of transition between the different states and the five clusters

\section{Simulation of the effect of treatment}

In the simulations we assumed that $67 \%$ of the LBP episodes benefited from treatment, based on data from the literature [24]. We hypothesized that the pain trajectories described above (Fig. 1) referred to the natural history of LBP, in absence of any treatment. The trajectories of the five clusters were therefore used to model the behaviour of the pain score in LBP episodes which did not receive treatment. For episodes receiving treatment, a time-dependent component modelling its effect was subtracted from the score describing the pain trajectory; this component had an initial peak in effectiveness at one month after the beginning of the treatment followed by a progressive decrease in the subsequent weeks (Fig. 3), simulating a stronger effect in the first stages of the pain episode blending into the natural trajectory of pain one year after the onset. The treatment randomly began on any given day during the first 30 days after the onset 
Table 1 Probabilities of remaining in the same cluster, transitioning to another cluster or terminating the episode after 12 weeks (for clusters 1 and 2) and one year (for clusters 3, 4, 5). Data estimated from [23]

\begin{tabular}{|c|c|c|c|c|c|}
\hline \multicolumn{6}{|c|}{ Cluster in the simulated 12 weeks/year } \\
\hline Cluster in the following year & 1: rapid recovery & $\begin{array}{l}2: \text { recovery at } \\
\text { week } 12\end{array}$ & $\begin{array}{l}\text { 3: incomplete recovery/ } \\
\text { persistent mild pain }\end{array}$ & 4: fluctuating pain & $\begin{array}{l}\text { 5: persis- } \\
\text { tent high } \\
\text { pain }\end{array}$ \\
\hline Episode termination & $99.4 \%$ & $99.4 \%$ & $0.4 \%$ & - & - \\
\hline $\begin{array}{l}\text { 3: incomplete recovery/persistent } \\
\text { mild pain }\end{array}$ & $0.6 \%$ & $0.6 \%$ & $98.6 \%$ & $4.4 \%$ & $0.4 \%$ \\
\hline 4: fluctuating pain & - & - & $1 \%$ & $94.8 \%$ & $1 \%$ \\
\hline 5: persistent high pain & - & - & - & $0.8 \%$ & $98.6 \%$ \\
\hline
\end{tabular}

Fig. 3 Effect of treatment on the pain score for episodes in clusters 1 and 2 (left), and clusters $3,4,5$ (right). The continuous lines represent the mean value over all episodes, whereas the error bars represent the standard deviations which were used to randomly create the individual curves associated with each episode. In the model, the effect of treatment is subtracted from the curve describing the agentspecific pain trajectory
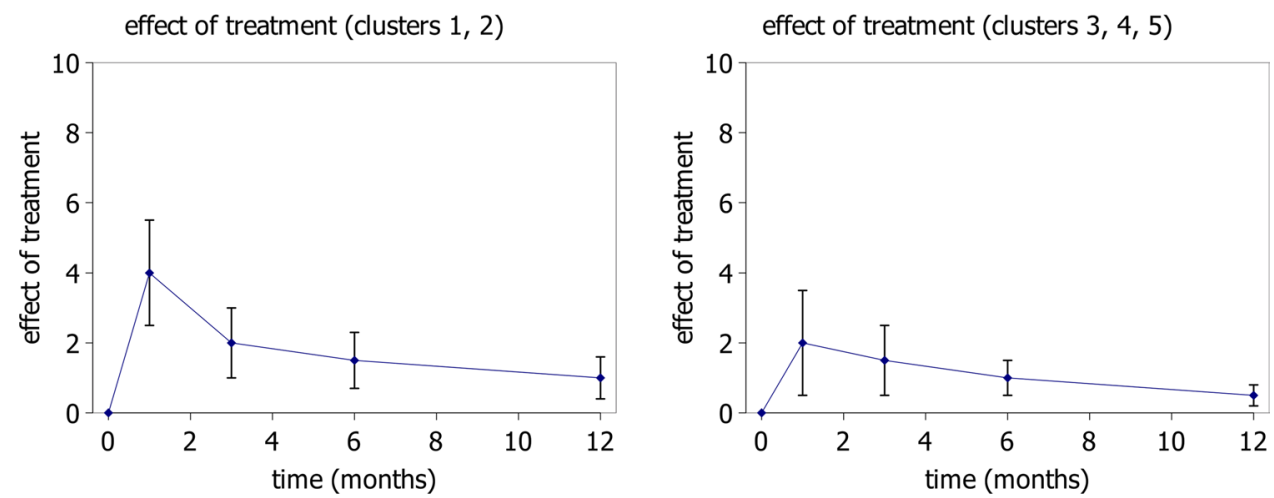

of the pain episode. The magnitude of the treatment effect was dependent on the cluster of trajectories of LBP; clusters 1 and 2 showed a higher initial peak in effectiveness, whereas the curve was flatter for episodes of persistent pain (clusters 3, 4, 5). A random variability was introduced to replicate the different response of individual patients to the treatment. Due to the lack of epidemiological data, the shape and amplitude of the curves modelling the effect of treatments were based on the personal experience of the authors.

\section{Simulation of reduced access to treatment during lockdown}

Due to the lack of quantitative data and the large variability of lockdown policies among countries, the impact of the reduced access to treatment was estimated by means of a probabilistic approach. Starting from the population at the steady state, 500 distinct scenarios described by five randomly estimated variables determining the impact of the lockdown were simulated. The five variables were as follows: (1) the length of the lockdown (lck_len); (2) the probability of having access to treatment for an incident episode of LBP during the lockdown, or of continuing treatment for episodes which started before the lockdown (lck_prob); (3) the maximal number of days between the end of the lockdown and the resumption of treatment (days_restart); (4) the average duration of beneficial effects of the treatment after its interruption, for episodes belonging to clusters 1 and 2 (days_lost_1_2); (5) the same duration for episodes in clusters 3, 4, and 5 (days_lost_3_4_5) (Table 2).

The values of the variables for the 500 scenarios were drawn from a uniform statistical distribution. All simulations covered a period of 2 years starting from the first day of the lockdown. The three variables days_restart, days_lost_1_2 and days_lost_3_4_5 described the range of allowed values for the whole population, whereas each individual had a distinct response to treatment which was randomly determined. The interruption of the treatment impacted the pain intensity by progressively reducing the benefit in a random timeframe between 1 day, thus modelling an immediate loss of the effect of treatment, and days_lost_1_2 (for episodes in clusters 1 and 2) or days_lost_3_4_5 (for episodes in clusters 3, $4,5)$. Individuals who were undergoing treatment before the interruption resumed their therapy according to a randomly determined number of days between 1 and days_restart.

In addition to the simulation study, we simulated two representative scenarios in which the access to treatment was reduced by 30 and 90 days, respectively. In both scenarios, $l c k \_$prob was set to 0.1 , i.e. $10 \%$ of episodes had access to treatment during the lockdown; days_restart was set to 60 , i.e. the agents which had access to treatment before the lockdown resumed it within the 2 months following the end 
Table 2 Name, description, ranges of the values of the five random variables in the probabilistic study, and their values used in the two representative scenarios

\begin{tabular}{|c|c|c|c|}
\hline Variable & Description & Simulated values & $\begin{array}{l}\text { Repre- } \\
\text { sentative } \\
\text { scenarios }\end{array}$ \\
\hline lck_len & Length of the lockdown (days) & $15-120$ & 30,90 \\
\hline$l c k \_p r o b$ & $\begin{array}{l}\text { Probability of having access to treatment for episodes having onset during the lockdown, or of } \\
\text { continuing the treatment for episodes which started before the lockdown (-) }\end{array}$ & $0-0.4$ & 0.1 \\
\hline days_restart & $\begin{array}{l}\text { Maximal number of days between the ending of the lockdown and the resumption of treat- } \\
\text { ment (days) }\end{array}$ & $15-730$ & 60 \\
\hline days_lost_1_2 & $\begin{array}{l}\text { Average length of the period before the loss of the beneficial effects of the treatment after its } \\
\text { suspension (clusters } 1,2 \text { ) (days) }\end{array}$ & $3-45$ & 30 \\
\hline days_lost_3_4_5 & $\begin{array}{l}\text { Average length of the period before the loss of the beneficial effects of the treatment after its } \\
\text { suspension (clusters } 3,4,5 \text { ) (days) }\end{array}$ & $30-20$ & 60 \\
\hline
\end{tabular}

of the lockdown; days_lost_1_2 was set to 30 and days_ lost_3_4_5 to 120, thus modelling a slower loss of the treatment benefits for individuals suffering from persistent pain.

\section{Data analysis and metrics}

The accuracy of the model in simulating LBP in the general population was evaluated by comparing its incidence with existing epidemiological data $[3,20]$. The impact of the lockdown was first assessed by comparing the evolution of the average pain intensity score in the two simulated scenarios with a baseline scenario in which lockdown was not modelled. The metrics which were used for the comparison were: (1) the percentage of individuals with a pain intensity score greater than 0 but lower than 3 ; (2) the percentage of individuals with a pain intensity score greater than 3 , which would likely be associated with disability [3]; (3) the average score of all individuals with LBP; (4) the score of the individuals belonging to each cluster.

The simulation study allowed for the calculation of the relative importance of the five random variables, namely lck_len, lck_prob, days_restart, days_lost_1_2, days_ lost_3_4_5, in determining a set of relevant outcomes describing the impact of the lockdown on the prevalence and severity of LBP in the population. The outcomes were calculated by comparing each simulation with the baseline scenario in which no lockdown was modelled, and were defined as follows: (1) the maximal increase, throughout the simulated period, of the prevalence of individuals with pain intensity score greater than 3 ; (2) the maximal increase of the pain intensity score for the individuals suffering from LBP, as well as for those belonging to each cluster; (3) the duration of the effects of the lockdown in terms of LBP pain intensity score, for all individuals suffering from LBP and specifically for each cluster. The latter outcome was estimated as the base of the triangle with area equal to that between the curves referring to the score of the baseline simulation and the simulation of interest, and height equal to the maximal increase of the score with respect to the baseline (Fig. 4). For each of these outcomes, the relative importance of the five random variables was estimated by means of a gradient boosting regression model $[25,26]$. In addition, after checking the non-normality of the distributions, Kruskal-Wallis tests were performed to assess the differences in the metrics among all individuals with LBP and those belonging to the five clusters. In case of significance, Wilcoxon rank-sum tests with Bonferroni correction to account for multiple comparisons were then performed to determine the level of significance for the differences between the groups. All statistical calculations were performed by means of the Python libraries SciPy (https:// www.scipy.org/) and scikit-learn (https://scikit-learn.org). Data plots were created with Matplotlib (https://matplotlib. org) and Seaborn (https://seaborn.pydata.org).

\section{Results}

The simulation of LBP in the general population revealed that approximately $4 \%$ of individuals had a pain intensity score between 0 and 3 , whereas the prevalence of scores greater than 3 was $2.5 \%$ (Fig. 5). These results agree with the global prevalence of LBP of $7.5 \%$ calculated for 2017 based on data from the Global Burden of Disease, Injuries, and Risk Factors Study [3].

The reduced access to treatment due to lockdown in the two representative scenarios induced an increase of $1 \%$ for the prevalence of individuals with score greater than 3 with respect to the baseline case with no lockdown, which was compensated by a corresponding decrease in the individuals with score between 0 and 3 . These scenarios showed only small differences, with marginally higher prevalence of individuals with scores greater than 3 for the scenario with a lockdown duration of 90 days (Fig. 5). Approximately one 
Fig. 4 Method for the estimation of the duration of the effects of the lockdown, as the base of the triangle with area (in blue) equal to that between the curves referring to the score of the baseline simulation (in red) and the simulation of interest (in green), and height equal to the maximal increase of the score with respect to the baseline
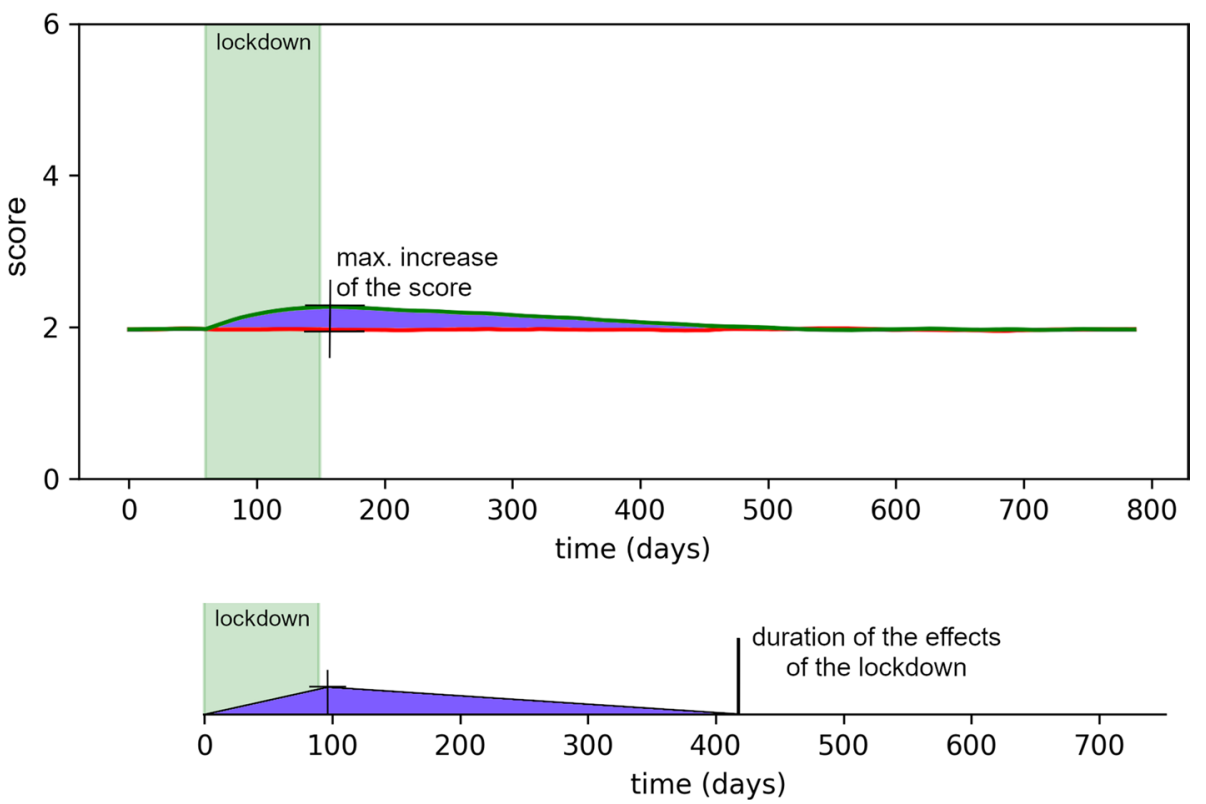

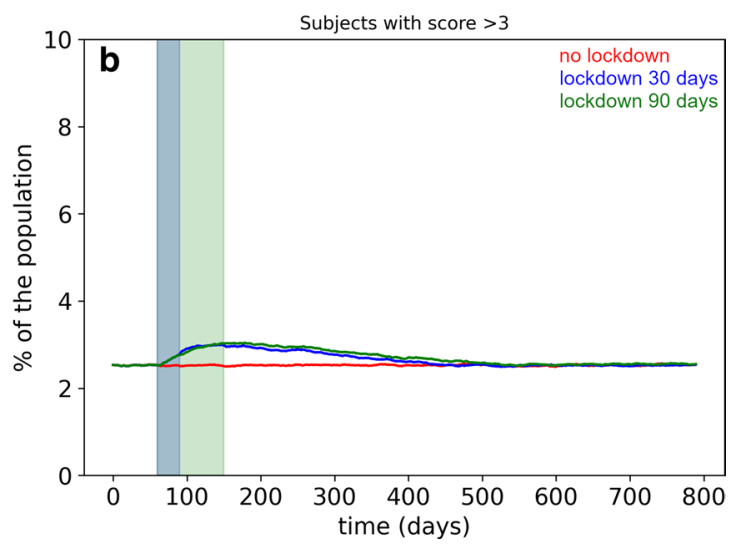

representative scenarios with lockdown durations of 30 days (blue) and 90 days (green)

to treatment. Cluster 1 showed a behaviour distinct from all other clusters; markedly higher pain scores were predicted for the scenario with the longer lockdown, and the effects of the lockdown ceased within two months after treatment resumption.

The simulation study revealed that the maximal increase in the score for individuals belonging to clusters 3,4 , and 5 as well as for all those suffering from LBP were not statistically different and had a mean value of 0.25 (Fig. 7a, c). Clusters 1 and 2, however, showed a distinct behaviour, with mean score increases of 0.9 and 0.7 , respectively, as well as a larger statistical distribution. In contrast, the estimated length of the effects of the lockdown showed statistically significant differences among all groups (Fig. 7b, d). For cluster 1 , the mean length was 86 days, whereas for all other clusters completely one year after the resumption of the full access 

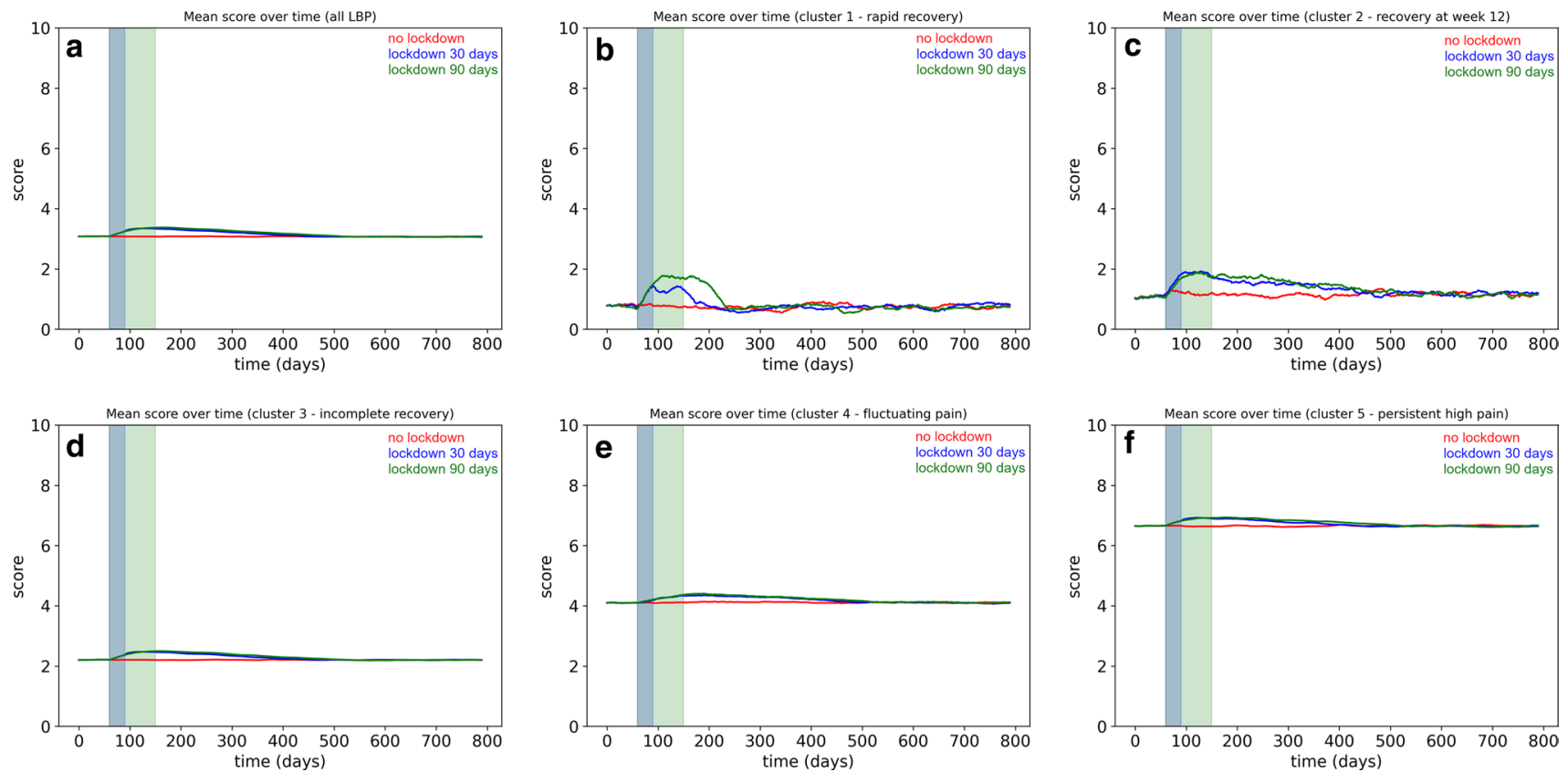

Fig. 6 Mean pain score over two years for all individuals suffering from LBP (a) and for individuals belonging to each cluster (b-f), predicted for the baseline case in which no lockdown was simulated (red) as well as for the two representative scenarios with lockdown durations of 30 days (blue) and 90 days (green)

pain in the population lasts more than one year, especially in case of persistent or fluctuating pain. Nevertheless, this could lead to an increase in disability for individuals with pain $\geq 3$, or to the onset of disability with those with milder pain. It should be noted that the reported changes in the pain score represent the average of a population, whereas each individual may suffer from higher increases up to 4-5 points for episodes in clusters 1 and 2 .

The analysis of the probabilistic data revealed that all the five randomly created variables (lck_len, lck_prob, days_ restart, days_lost_1_2, days_lost_3_4_5) had, although in different terms with respect to the trajectories of pain, a relevant impact in determining both the increase of pain intensity in the population and the length of the effects of the lockdown. Therefore, the model confirmed that the impact of the containment measures cannot simply be minimized by acting on one individual variable, such as for example the length of the lockdown, because other determinants related to the lockdown influenced the outcomes. We could not therefore identify the "optimal lockdown parameters" which would minimize the impact on LBP while preserving the general effects on infection spread and mortality [28]. However, the variable days_restart seemed to have an important role in determining the maximal increase of the pain score and the duration of the effects of the lockdown. This implies that policies aiming at favouring a prompt resumption of the evidence-based treatment after the lockdown may be effective in shortening the duration of its negative effects. 

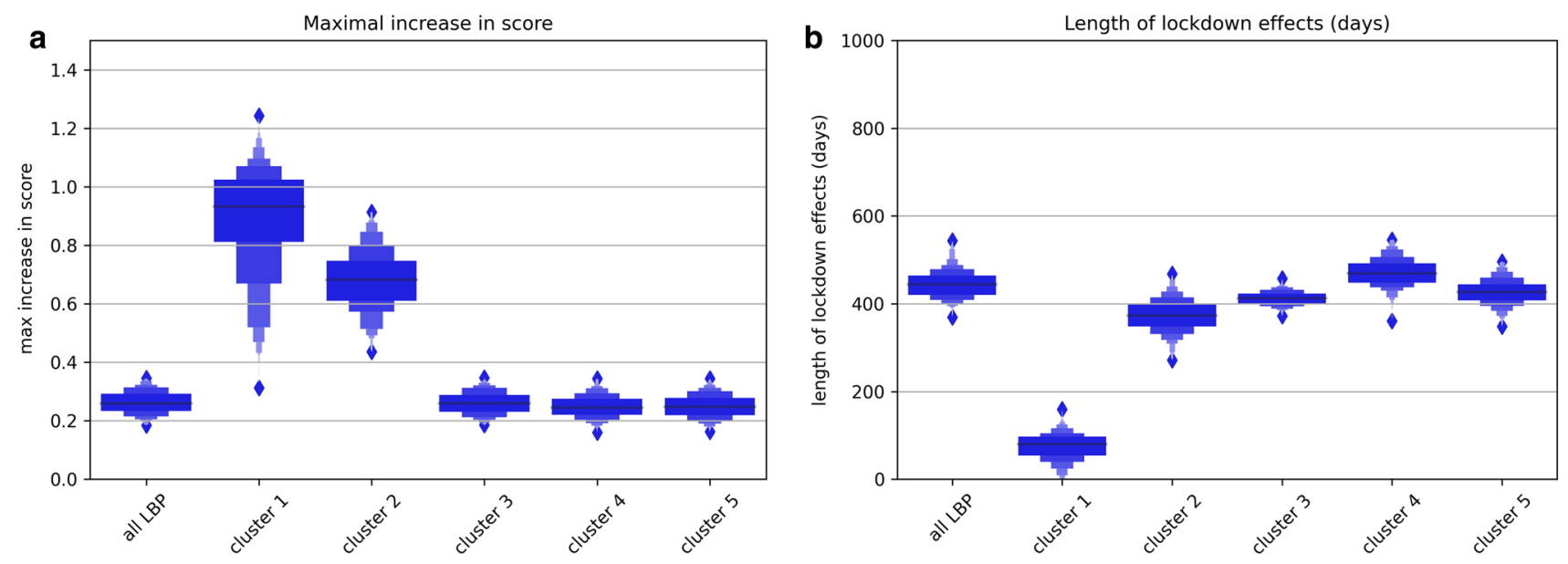

C Maximal increase in score
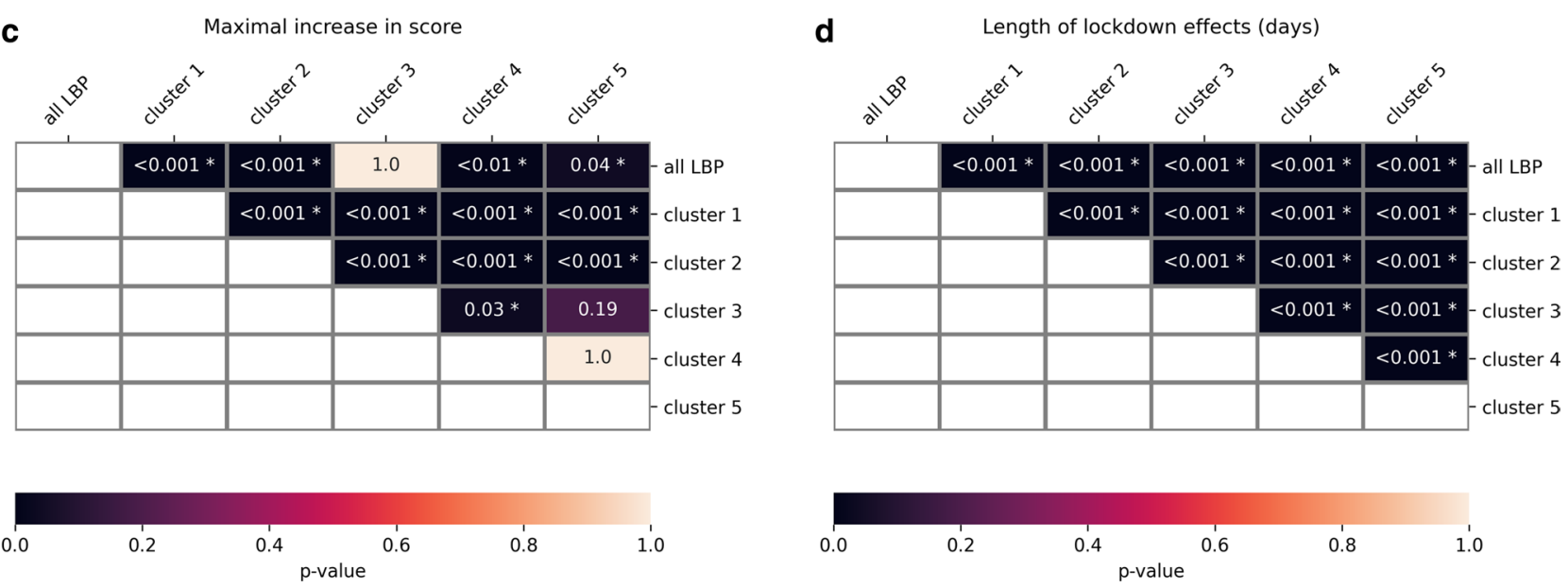

Fig. 7 Statistical distribution of the maximal increase in the score (a) and of the estimated length of the effects of the lockdown (b) for all individuals suffering from LBP ("all LBP") and for those belonging

Alternatively, it could suggest that providing patients with self-management strategies may assist in mitigating the impact of the lockdown on LBP, although this could not be proven within this study in which the effect of such strategies was not modelled.

The findings of our paper may provide a basis for the estimation of the burden of the containment measures for COVID-19 with respect to some aspects which have been neglected so far, such as the delayed return to work for patients suffering from LBP and the increased healthcare costs associated with the generally higher prevalence and severity of the disease. Although the benefits of the lockdown in terms of reduction of the infection rate, mortality, and admissions to hospitals and intensive care units [29, 30] have evidently a higher degree of priority in the management of the COVID-19 pandemic, the lockdown and the consequent reduced access to treatments may indeed have serious implications on public health [31-33], and appropriate measures to minimize them may need to be taken. Therefore,

to the five clusters. Heatmaps and p-values of the statistical comparisons between groups regarding the maximal increase in score (c) and length of the effects of the lockdown (d)

estimating the impact of the containment measures, and of the specific aspects of the measures determining such an impact, constitutes the first, fundamental step for taking effective decisions, and mathematical models may play a critical role in this respect.

To our knowledge, this paper presents the first agent-based model aimed at investigating LBP in the general population. The results about the prevalence of LBP in the general population (4\% with a pain intensity score between 0 and 3, and $2.5 \%$ with score greater than 3) agree with the global prevalence of LBP of 7.5\% calculated for 2017 based on data from the Global Burden of Disease, Injuries, and Risk Factors Study [3]. Although the findings generally agree with available data, the modelling approach suffers from some limitations typically associated with a new methodology to understand new problems, as well as with the incompleteness of the data available in the literature with respect to the data which would be theoretically necessary for the establishment and validation of such a model [34]. Although the incidence and pain trajectories of 

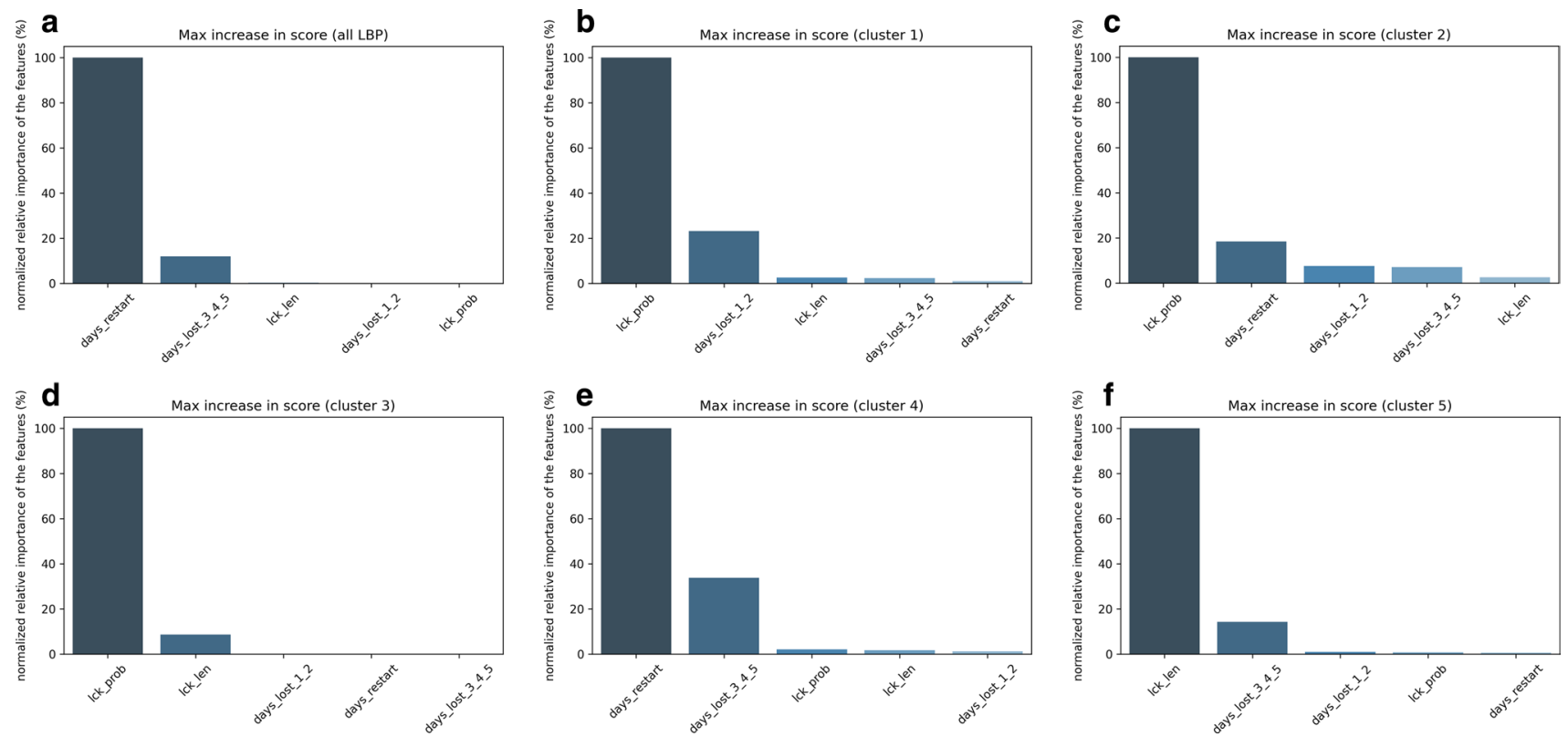

Fig. 8 Relative importance of the five random variables (lck_len, lck_prob, days_restart, days_lost_1_2, days_lost_3_4_5) in determining the maximal increase of the score for all individuals suffering from LBP (a) as well as for the individuals belonging to the five clusters (b-f)
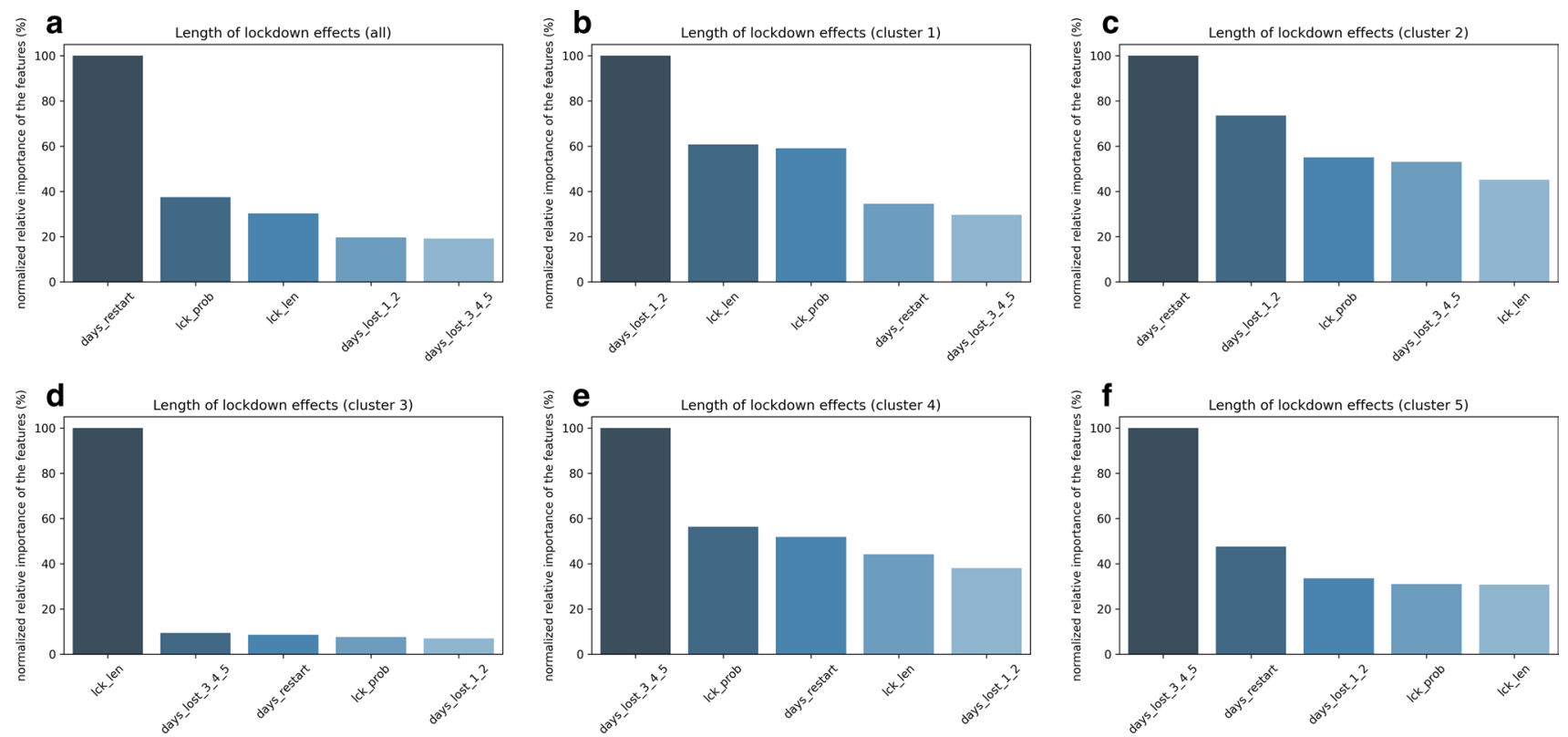

Fig. 9 Relative importance of the five random variables (lck_len, lck_prob, days_restart, days_lost_1_2, days_lost_3_4_5) in determining the length of the effects of the lockdown for all individuals suffering from LBP (a) as well as for the individuals belonging to the five clusters (b-f)

the disease were based on large-scale studies [20, 22, 23], we lack data about some relevant aspects and we had to make assumptions. For example, data about the impact of the access to treatment could not be found, and the differences in the pain intensity score between patients with or without access to treatment (Fig. 3) were therefore estimated based on the personal experience of the authors. Furthermore, we assumed that the available data about the trajectories of pain (Fig. 1) was relevant to individuals without access to treatment, even though these estimates were obtained from samples that included participants who received treatment for their LBP [22]. Moreover, no data is available to describe the impact of the lockdown on the access to treatment for LBP. In addition, parameters such as the duration of the lockdown are known, but show a large 
geographical variability [35] which makes difficult their proper representation in a simulation; indeed, the current model does not represent any specific country but is based on data from sources located in different continents. In order to mitigate this lack of data and the regional variability, we decided to employ a probabilistic approach in which the values of the unknown quantities were not fixed by means of a reasonable guess but were randomized in a large number of hypothetical scenarios. This approach allowed for the quantification of the importance of each of these unknown quantities by means of a standard statistical method like gradient boosting regression modelling. Finally, the modelling approach did not take into account the interactions between LBP and comorbidities, such as for example depression and anxiety which are commonly co-occurring with chronic pain and disability [36, 37], and other psychological implications such as fear-avoidance behaviour and anticipation of pain. It should be noted that not explicitly modelling quantities such as anxiety and depression does not imply neglecting their effects on the general population, which are captured by the trajectories of pain for the five clusters (Fig. 1). Besides, a further extension of the modelling approach to consider also such aspects would have necessarily required additional assumptions, due to the general incompleteness of the available data mentioned above.

Aside from the limitations associated with the lack of data and assumptions, the agent-based model consisted of a number of agents, 300,000, which is typically lower than the population of most countries and regions. However, preliminary tests revealed that increasing the number of agents would have a minimal impact on the results of the simulations in terms of prevalences and pain scores. Regarding the simulation of treatment, we did not make a distinction between the different options which may be distinctly impacted by the lockdown; whereas rehabilitation interventions are indeed largely affected [2], access to pharmacological treatments such as non-steroidal anti-inflammatory drugs may not considerably be impeded. It should also be noted that this study does not fully exploit the potential of agent-based models, which are especially powerful when used to simulate agents that interact with each other, for example transmitting an infectious disease. Due to the nontransmissible nature of low back pain and rehabilitation interventions, interactions between agents are not relevant for this study, which cannot therefore take full advantage of the potential of the method.

\section{Conclusions}

In summary, this study achieved two major goals. First, we established the first agent-based model of LBP in the general population, able to replicate the prevalence of LBP described in epidemiological studies as well as the distribution of pain trajectories in the simulated individuals. Second, we found a prevalent effect of the lockdown on the length of pain episodes (up to one year), which may impact on the rate of chronicity and disability. The quantitatively estimated impact on pain intensity is small and below the MCID, but it lasts for a long time period, exceeding one year thus having a potential large impact in terms of disability. We believe that agent-based modelling constitutes a powerful tool for the study of public healthcare, and may help the understanding of major global challenges like LBP as well as the impact of events such as pandemics on the general population.

Funding The study has been partially funded by the Italian Ministry of Health ("Ricerca Corrente").

\section{Declaration}

Conflict of interest The authors have no conflicts of interest to declare.

Availability of data and material The data that support the findings of this study are available from the corresponding author, upon reasonable request. The code is available from the corresponding author, upon reasonable request.

\section{References}

1. Palmer K, Monaco A, Kivipelto M, Onder G, Maggi S, Michel JP, Prieto R, Sykara G, Donde S (2020) The potential long-term impact of the COVID-19 outbreak on patients with non-communicable diseases in Europe: consequences for healthy ageing. Aging Clin Exp Res 32:1189-1194. https://doi.org/10.1007/ s40520-020-01601-4

2. Negrini S, Grabljevec K, Boldrini P, Kiekens C, Moslavac S, Zampolini M, Christodoulou N (2020) Up to 2.2 million people experiencing disability suffer collateral damage each day of Covid-19 lockdown in Europe. Eur J Phys Rehabil Med 56(3):361-365. https://doi.org/10.23736/S1973-9087.20.06361-3

3. Wu A, March L, Zheng X, Huang J, Wang X, Zhao J, Blyth FM, Smith E, Buchbinder R, Hoy D (2017) Global low back pain prevalence and years lived with disability from 1990 to 2017: estimates from the Global Burden of Disease Study 2017. Ann Transl Med 8:299

4. Hoy D, Bain C, Williams G, March L, Brooks P, Blyth F, Woolf A, Vos T, Buchbinder R (2012) A systematic review of the global prevalence of low back pain. Arthritis Rheum 64:2028-2037

5. Maher C, Underwood M, Buchbinder R (2017) Non-specific low back pain. Lancet 389:736-747

6. Hartvigsen J, Hancock MJ, Kongsted A, Louw Q, Ferreira ML, Genevay S, Hoy D, Karppinen J, Pransky G, Sieper J, Smeets RJ (2018) What low back pain is and why we need to pay attention. Lancet 391:2356-2367

7. Nurchis MC, Pascucci D, Sapienza M, Villani L, D’Ambrosio F, Castrini F, Specchia ML, Laurenti P, Damiani G (2020) Impact of the burden of COVID-19 in Italy: results of Disability-Adjusted Life Years (DALYs) and productivity loss. Int J Environ Res Public Health. https://doi.org/10.3390/ijerph17124233

8. Newman KB, Buckland ST, Morgan BJT, King R, Borchers DL, Cole DJ (2014) Modelling population dynamics: model 
formulation, fitting and assessment using state-space methods. Springer, New York

9. Matsuda H, Ogita N, Sasaki A, Satō K (1992) Statistical mechanics of population: the lattice Lotka-Volterra model. Progr Theoret Phys 88:1035-1049

10. Kermack WO, McKendrick AG, Walker GT (1927) A contribution to the mathematical theory of epidemics. P R Soc Lond Ser A Contain P Math Phys Character 115: 700-721

11. Situngkir H (2004) Epidemiology through cellular automata: case of study avian influenza in Indonesia. arXiv. http://arxiv.org/abs/ nlin/0403035

12. Tang J, Fernandez-Garcia I, Vijayakumar S, Martinez-Ruis H, Illa-Bochaca I, Nguyen DH, Mao JH, Costes SV, Barcellos-Hoff MH (2014) Irradiation of juvenile, but not adult, mammary gland increases stem cell self-renewal and estrogen receptor negative tumors. Stem Cells 32:649-661

13. Tang J, Ley KF, Hunt CA (2007) Dynamics of in silico leukocyte rolling, activation, and adhesion. BMC Syst Biol 1:14

14. Allen TT (2011) Introduction to discrete event simulation and agent-based modeling: voting systems, health care, military, and manufacturing. Springer Science \& Business Media, Berlin

15. Silverman BG, Hanrahan N, Bharathy G, Gordon K, Johnson D (2015) A systems approach to healthcare: agent-based modeling, community mental health, and population well-being. Artif Intell Med 63:61-71

16. McLean A, McDonald W, Goodridge D, Osgood N (2019) Agentbased modeling: a method for investigating challenging research problems. Nurs Res 68:473-482

17. US Census Bureau. Estimates of U.S. Population by Age and Sex. https://www.census.gov/newsroom/press-releases/2018/pop-chara cteristics.html

18. Life Expectancy Data Viz (2020). https://www.cdc.gov/nchs/datavisualization/life-expectancy/

19. Edwards RD (2008) The cost of uncertain life span. National Bureau of Economic Research. http://www.nber.org/papers/ w14093

20. Cassidy JD, Côté P, Carroll LJ, Kristman V (2005) Incidence and course of low back pain episodes in the general population. Spine 30:2817-2823

21. Côté P, Cassidy JD, Carroll L (2001) The treatment of neck and low back pain: who seeks care? who goes where? Med Care. JSTOR 39:956-967

22. Downie AS, Hancock MJ, Rzewuska M, Williams CM, Lin C-WC, Maher CG (2016) Trajectories of acute low back pain: a latent class growth analysis. Pain 157:225-234

23. Chen Y, Campbell P, Strauss VY, Foster NE, Jordan KP, Dunn KM (2018) Trajectories and predictors of the long-term course of low back pain: cohort study with 5-year follow-up. Pain 159:252-260

24. Beyera GK, O'Brien J, Campbell S (2019) Health-care utilisation for low back pain: a systematic review and meta-analysis of population-based observational studies. Rheumatol Int 39:1663-1679

25. Friedman JH (2001) Greedy function approximation: a gradient boosting machine. Ann Stat 29:1189-1232

26. Friedman JH (2002) Stochastic gradient boosting. Comput Stat Data Anal 38:367-378

27. Von Korff M, Jensen MP, Karoly P (2000) Assessing global pain severity by self-report in clinical and health services research. Spine 25:3140-3151

28. Gonzalez-Eiras M, Niepelt D (2020) On the optimal lockdown during an epidemic. https://www.econstor.eu/handle/10419/ 216636

29. Salje H, Kiem CT, Lefrancq N, Courtejoie N, Bosetti P, Paireau J, Andronico A, Hozé N, Richet J, Dubost CL, Le Strat Y (2020) Estimating the burden of SARS-CoV-2 in France. Science 369(6500):208-211. https://doi.org/10.1126/science.abc3517

30. Roux J, Massonnaud C, Crépey P (2020) COVID-19: One-month impact of the French lockdown on the epidemic burden. medRxiv https://www.medrxiv.org/content/https://doi.org/10.1101/2020.04. 22.20075705v1.abstract

31. Thornton J (2020) Covid-19: A\&E visits in England fall by $25 \%$ in week after lockdown. BMJ 369. https://www.bmj.com/content/ 369/bmj.m1401.long

32. Zhao J, Rudd A, Liu R (2020) Challenges and potential solutions of stroke care during the coronavirus disease 2019 (COVID-19) outbreak. Stroke Am Heart Assoc 51:1356-1357

33. Holmes JL, Brake S, Docherty M, Lilford R, Watson S (2020) Emergency ambulance services for heart attack and stroke during UK's COVID-19 lockdown. Lancet 395:e93-e94

34. Xiang X, Kennedy R, Madey G, Cabaniss S (2005) Verification and validation of agent-based scientific simulation models. Agentdirected Simul Conf 2005:55

35. Hale T, Petherick A, Phillips T, Webster S (2020) Variation in government responses to COVID-19. Blavatnik School of Government Working Paper. 31. https://www.bsg.ox.ac.uk/sites/default/ files/2020-04/BSG-WP-2020-032-v5.0_0.pdf

36. Pinheiro MB, Ferreira ML, Refshauge K, Colodro-Conde L, Carrillo E, Hopper JL, Ordoñana JR, Ferreira PH (2015) Genetics and the environment affect the relationship between depression and low back pain: a co-twin control study of Spanish twins. Pain 156:496-503

37. Marshall PWM, Schabrun S, Knox MF (2017) Physical activity and the mediating effect of fear, depression, anxiety, and catastrophizing on pain related disability in people with chronic low back pain. PLoS ONE 12:e180788

Publisher's Note Springer Nature remains neutral with regard to jurisdictional claims in published maps and institutional affiliations. 